NBER WORKING PAPER SERIES

INVESTMENTS OF UNCERTAIN COST

Robert S. Pindyck

Working Paper No. 4175

\author{
NATIONAL BUREAU OF ECONOMIC RESEARCH \\ 1050 Massachusetts Avenue \\ Cambridge, MA 02138 \\ September 1992
}

This research was supported by the M.I.T, Center for Energy Policy Research, and by the National Science Foundation under Grant No. SES-8618502. My thanks to Alexardar Angelus and David Chariton for their excellent research assistance, to Burbara MacMullan and Lewis Perl of NERA for making availabie their nuclear power plant cost data and regressions, and to John Cox, Geste Grossman, Paul Joskow, Richard Lester, Lewis Perl, William Pounds, Martin Weizman and an anonymous referee for belpful comments and suggestions. This paper is part of NBER's research programs in Industrial Organization and Productivity. Any opinions expressed are those of the author and not those of the National Bureau of Economic Research. 
NBER Working Paper \#4175

September 1992

\section{INVESTMENTS OF UNCERTAIN COST}

\section{ABSTRACT}

I study irreversible investment decisions when projects take time to complete, and are subject to two types of uncertainty over the cost of completion. The furst is technical uncertainty, i.e., uncertitinty over the amount of time, effort, and materials that will ultimately be required to complete the project, and that is only resolved as the investment proceeds. The second is input cost uncerainty, ic, uncertainty over the prices and quantities of labor and materials required, and which is external to the firm's inyestment activity. I derive a simple decision rule that maximizes the fim's value, and I use it to show how these two types of uncertainty have very different effects on investment decisions. As an example, I analyze the decision to start or continue building a nuclear power plant during the 1980 's.

Robert S. Pindyck

MIT

Cambridge, MA 02139

and NBER 


\section{Introduction.}

In most studies of investment under uncertainty, it is the future payoffs from the investment that are uncertain. The emphasis on uncertainty over future payoffs also applies to the growing literature on irreversible investment. Much of that literature (see Dixit (1992) and Pindyck (1991) for an overview) studies optimal stopping rules for the timing of sunk costs of known magnitude, in exchange for capital whose value fluctuates stochastically.

At times the cost of an investment is more uncertain than the future payoff. This is olten the case for large projects that take considerable time to build. An example is a nuclear power plant, where total construction costs are hard to predict due to both engineering and regulatory uncertainties. Although the future value of a completed nuclear plant is also uncertain (because electricity demand and costs of alternative fuels are uncertain), construction cost uncertainty is much greater, and has deterred utilities from building new plants. There are many other examples, ranging from large petrochemical complexes, to the development of a new line of aircraft, to urban construction projects. Also, large size is not a requisite. Most $\mathrm{R} \& \mathrm{D}$ projects involve considerable cost uncertainty; the development of a new drug by a pharmaceutical company is an example.

In addition to their uncertain costs, all of the investments mentioned above are irreversiole. Expenditures on nuclear power plants, petrochemical complexes, and the development of new drugs are firm- or industry-specific, and hence are sunk costs that cannot be recovered should the investment turn out, ex post, to have been a bad onc. In each case, the investment could turn out to be bad because demand for the product is less than anticipated, or because the cost of the investment turns out to be greater than anticipated. Whatever the reason, the firm cannot "disinvest" and recover the money it spent.

This paper studies the implications of cost uncertainty for irreversible investment decisions. I am concerned with projects that take time to complete, so that two different kinds of uncertainty arise. The first, which I call technical uncertainty, relates to the difficulty of completing a project: Assuming prices of construction inputs are known, how much time, effort, and materials will ultimately be required? This kind of uncertainty can only be resolved by 
undertaking the project; actual costs and construction time unfoid as the project proceeds. ${ }^{1}$ These costs may from time to time be greater or less than anticipated as impediments arise or as the work progresses faster than planned, but the total cost of the investment is only known for certain when the project is complete. Also, this uncertainty is largeiy diversifiable. It results only from the inability to predict how diffcult a project will be, which is likely to be independent of the overall economy.

The second kind of uncertainty relates to input cosis, and is external to what the firm does. It arises when the prices of labor, land, and materials needed to build a project fluctuate unpredictably, or when unpredictable changes in govenment regulations change the required quantities of construction inputs. Prices and regulations change whether of not the firm is investing, and are more uncertain the farther into the future one looks. Hence input cost uncertainty is particularly important for projects that take time to complete, or are subjact to voluntary or involuntary delays. Also, this uncertainty may be partly nondiversifiable; changes in construction costs are likely to be correlated with overall economic activity.

This paper derives decision rules for irreversible investments subject to both types of cost uncertainty. For simplicity, I first assume that the value of the completed project is known with certainty, and then show how the model can be extended so that this is also stochastic. The decision rules I derive allow for the possibility of abandoning the project midstream, and maximize the value of the firm in a competitive capital market. These rules have a simple form: Invest as long the expected cost to complete the project is below a aritical number. Also, the derivation of the decision rule yields the value of the investment opportunity, i.e., what one would pay for the right to undertake the project. I explore how this value, and the critical expected cost to completion, depend on the type and level of uncertainty.

Both types of uncertainty increase the value of an investment opportunity. The reason is that the payoff function is $\max [0, V-K]$, where $K$ is the cost and $V$ the value of the completed project. The investment opportunity is like a put option; the holder can sell an

\footnotetext{
${ }^{1}$ This is a simplification, in that for some projects cost uncertainty can be reduced by first undertaking additional engineering studies. The investment problem is then more complicated because one has three choites instead of two: start construetion now, undertake an engineering sludy and then begin construction only if the study indicates costs are likely to be low, or abandon the project complelely.
} 
asset worth an uncertain amount $K$ for a fixed "exercise price" $V$. Like any option, its value is increased by an increase in the variance of the price of the underlying asset. ${ }^{2}$

However, the two types of uncertainty affect the investment decision differently. Technical uncertainty raises the critical expected cost to completion. Hence a project can have an expected cost that makes its conventional NPV negative, but it can still be economical to begin investing. The reason is that investing reveals information about cost, and therefore has a shadow value beyond its direct contribution to the completion of the project; this shadow value lowers the full expected cost of the investment. ${ }^{3}$ Also, since information about cost arrives only when investment is taking place, there is no vaine to waiting.

As an example, a project requires a first phase investment of $\$ 1$. Then, with probability .5 the project will be finished, and with probability .5 a second phase costing $\$ 4$ will be required. Completion of the project yields a certain payoff of $\$ 2.8$. Since the expected cost of the project is $\$ 3$, the conventionally measured NPV is negative. But this ignores the value of the option to abandon the project should the second phase be required. The correct NPV is $-1+(.5)(2.8)=\$ 0.4$, so the firm should proceed with at least the first phase.

Input cost uncertainty reduces the critical expected cost. Hence a project could have a convectional NPV that is positive, but be uneconomical. This is because costs of construction inputs change whether or not investment is taking place, so there is a value of waiting for new information before committing resources. Also, this effect is magnified when fuctuations in construction costs are correlated with the economy, i.e, in the context of the CAPM, when the "beta" of cost is high. The reason is that a higher "beta" implies that high cost outcomes are more likely to be assocjated with high stock market returns, so that the investment opportunity is a hedge against nondiversifiable risk. Put another way, a higher "beta" raises the discount rate applied to expected future costs, which raises the value of the investment opportunity, as well as the benefit from waiting rather than investing now.

\footnotetext{
${ }^{2}$ Using put-call parity, we can also think of this as a call option with a stochastic execcise price $(K)$ on an asset with a fixed value $(V)$. In my model, the firm has a more complicated compound option; it can spend an uncertain amount of money in return for an option to continue the partially completed project.

${ }^{3}$ It is analogous to the shadow value of praduction arising from a learsing curve, which lowers the full cost of production; see Majd and Pindyck (1989).
} 
For example, suppose an investment can be made now or later. The cost is now 53 , but next period it will either fall to $\$ 2$ or rise to $\$ 4$, each with probability .5 , and then remain at that level. Investing yields a certain payoft of $\$ 3.2$, and assume the risk-free rate of interest is zero. If we invest now, the project has a conventional NPV of $\$ 0.2$. But this ignores the opportunity cost of closing our option to wait for a better outcome (a drop in cost). If we wait; we will only invest if the cost falls to $\$ 2$. The NPV if we wait is $(.5)(3.2-2)=\$ 0.6$, so it is beller to wait. Now suppose the "beta" of cost is high, so that the risk-adjusled discount rate is 25 percent per period. Because the payoff from completing the project is certain, this discount rate is only applied to cost. Hence the NPV if we wait is now (-5) [3.2 $-2 / 1.25]=80.8$. The higher "beta" raises the present vaiues of net payofs, and thereby increase both the value of the investment opporturity and the value of waiting-

This paper is related to several earlier studies. The value of inlormation gathering has been explored by Roberts and Weitzman (1981), who developed a model of sequential investment similar to mine in that the project can be stopped in midstream, and the process of investing reduces both the expected cost of completing the project as well the variance of that cost. They derive an optimal stopping rule, and show that it may pay to go ahead with the early stages of an investment even though the NPV of the entire project is negative." Grossman and Shapiro (1986) also study investments for which the total effort required to reach a payoff is unknown. They model the payoff as a Poisson arrival, with a hazard rate specifed as a function of the curnulative effort expended. They allow the rate of progress to be a concave function of effort, and locus on the rate of investment, rather than on whether one should proceed or not. My results complement those of these authors, but my model is more genera: in its treatment of cost uncertainty, and yjelds relatively simple decision rules.

This paper is also related to the basic model of irreversible investment by McDonald and Siegel (1986). They cousider the payment of a sunk cost $I$ in return for a project worth

\footnotetext{
${ }^{4}$ Weilzman, Newey, and Rabin (198I) use this model to evaluate demonstration plants for synthetic fuels, and show that learning about costs could justify these investments. Mackie-Mason (1991) extends the Roberts and Weitzman analysis by allowing for investors (who pay the cost of a project) and mangers (who decide whether to continue or abandon the project) to have conficting interests and asymmetric information. He shows that asymmetric learning about cost leads to inefficient overabandonment of projects. Finally, Zeira (1987) developed a model in which a fim learns about its payoft lunction as it accumulates capital.
} 
$V$, where $V$ and $I$ evolve as geometric Brownian motions. The optimal investment rule is to wait until $V / I$ reaches a critical value that exceeds 1 , because of the opportunity cost of committing resources. Also, Majd and Pindyck (1987) study sequential investment when a firm can invest at some maximum rate (so it takes time to complete a project), the project. can be abandoned before completion, and the value of the project, received upon completion, evolves as a geometric Brownian motion. In this paper the firm can also invest at a maximum rate, but it is the cost rather than the value of the completed project that is uncertain. ${ }^{5}$

The basic model is developed in the next section. In Section 3, numerical solutions are used to show how the value of the investment opportunity and the optimal investment rule depend on the source and amount of uncertainty, as well as other parameters. Section 4 analyzes the decision to build a nuclear power plant; it shows how the model can be used in practice, slows the importance of analyzing technical and input cost uncertainty together, and illustrates the nature and implications of nuclear plant cost uncertainty during the 1980's. Section 5 discusses some extensions of the basic model, and Section 6 concludes.

\section{The Basic Model.}

Consider an investment in a project whose actual cost of completion is a random variable, $\bar{K}$, and whose expected cost is $K=E(\tilde{K})$. The project takes time to complete; the maximum rate at which the firm can (productively) invest is $k$. Upon completion, the firm receives an asset (e.g., a factory or new drug) whose value, $V$, is known witl certainty.

If there were no uncertainty over the total cost, valuing the investmenl opportunity and determising the optimal investment rule would be straightforward. The project will take time $T=K / k$ to complete, so the opportunity to invest is worth:

$$
F(K)=\max \left[V e^{-r K / k}-\int_{0}^{K / k} k e^{-r t} d t, 0\right]
$$

\footnotetext{
${ }^{5}$ In reiated work, Baldwin (1982) artalyzes sequential jnvestment decisions when investment opportunilies arrive randomly and the firm has limited resources. She values the seçnence of opportunities and shows that a simple NPV rule leads to overinvestment, i.e., there is a value to waiting for better opportunities. Likewise, if cost evolvey stochastieally, it may pay to wait for cosi lo fall. Also, Myers and Majd (1984) determine the value of a firm's option to abandon a project in return for a scrap vaiue, $S$, when the value of the project, $V$, evolves as a geometric Brownian motion (the firm has a put option to sel] a project worth $V$ for a ptice $S$ ), and show how this abandonment value aflects the decision to inwest.
} 


$$
=\max \left[(Y+k / r) e^{-+K / k}-k / r, 0\right]
$$

where $r$ is the (risk-free) rate of interest. The optimal investment rule is to proceed with the project as long as $F(K)>0$, i.e, as long as $K$ is less than a critical $K$, given by:

$$
K^{=}=(k / r) \log (1+r V / k)
$$

If $T=0, F(K)=V-K$, and $K^{*}=V$. But if $\tau>0, F(K)<V-K$, and $K^{*}<V$, The reason is that the payof $V$ is recejved only at tine $T$, and must be discounted accordingly, but the cost of the investment is spread out from $t=0$ to $T$. Also, note that $F(K)$ is a convex function of $K$, so uncertainty over cost should increase $F(K)$. Little can be said at this point, however, about the effect of uncertainty on the optimal investment rule.

\section{Introducing Uncertainty.}

I introduce uncertainty over cost by letting the expected cost to completion, $K(t)$, lollow a controlled diffusion process. Suppose for the moment that $K(t)$ is given by:

$$
d K=-I d t+g(I, K) d z
$$

where $I$ is the rate of investment, $z(t)$ is a Wiener process that might or might rot be correlated with the economy and the stock market, and $g_{I} \geq 0, g_{i i} \leq 0$, and $g_{K} \geq 0$. Eqn. (2) says that the expected cost to go declines with ongoing investment, but also changes stochastically. Stochastic changes in $K$ might be due to technical uncertainty, in which case $g(0, K)=0$ and $g_{I}>0$, to input cost uncertainty, in which case $g(0, K)>0$, or to both."

I will again assume that there is a maximum rate of investment $k$. Let $F(K)=F(K ; V, k)$ be the value of the investment opportunity. Then $F(K)$ satisfies:

$$
F(K)=\max _{I(t)} E_{0}\left[V e^{-\mu \vec{T}}-\int_{0}^{\vec{T}} I(t) e^{-\mu t} d t\right]
$$

subject to eqn. $(2), 0 \leq I(t) \leq k$, and $K(\bar{T})=0$. Here $\mu$ is an appropriate rish-adjusted discount rate, and the time of completion, $\tilde{T}$, is stochastic.

\footnotetext{
${ }^{6} \mathrm{Eqn}$. (2) is a generalization of Roberts and Weitzman (1981), who also model the expected cost to go as a stochastic process that is controlled by the rate of investment.
} 
For eqn. (2) to make economic sense, more structure is needed. In particular, we wonld like: (i) $F(K, V, k)$ to be homogeneous of degree one in $K, V$, and $k$; (ii) $F_{K}<0$, i.e., an increase in the expected cost of an investment should always reduce its value; (iii) the instantaneous variance of $d K$ to be bounded for all finite $K$ and to approach 0 as $K \rightarrow 0$; and (iv) if the frrm invests at the maximum rate $k$ until the project is complete, $E_{0} \int_{0}^{\dot{T}} k d t=K$, so that $K$ is indeed the expected cost to completion. We can meet these conditions and still aliow for reasonably general cost structures by letting $g(I, K)=\beta K(I / K)^{\alpha}$, with $0 \leq \alpha \leq \frac{1}{2}$. This clearly satisfes conditions (i) and (iii). As will become evident later, $0 \leq \alpha \leq \frac{1}{2}$ rather than $0 \leq a<1$, which also satisfies (i) and (iii), is needed to satisfy (ii). Finally, it is shown in Appendix A that (iv) is also satisfied.

We wili restrict the analysis to $\alpha=0$ and $\frac{1}{2}$, which correspond naturally to our two types of cost uncertainty, and which result in simple corner solutions for optimal investment. (As discussed in Section 4, other values of $\alpha$ result in interior solutions where $I$ is varied in response to clanges in the variance of $\alpha K$.) The case of $\alpha=\frac{1}{2}$ corresponds to technical uncertaintyi $K$ can change only if the firm is investing, and the instantaneous variance of $d K / K$ increases linearly with $I / K$. When the firm is investing, the expected change in $K$ over an interval $\Delta t$ is $-I \Delta t$, but the realized change can be greater or less than this, and $K$ can even increase. As the project proceeds, progress will at times be slower and at times faster than expected. The variance of $\tilde{K}$ falls as $K$ falls, but the actual total cost of the project, $\int_{0}^{\bar{T}} I d t$, is only known when the project is completed.

The case of $\alpha=0$ corresponds to input cost uncertainty; the instantaneous variance of $d K / K$ is constant and independent of $I$. Now $K$ will fluctuate even when there is no investment; ongoing changes in the costs of labor and materials will change $K$ irrespective of what the firm does. And since the project takes time to build, the actual total cost of the project is again only known when the project is complete.

We can allow for both types of uncertainty by combining these two cases in a single equation for the evolution of $K$ :

$$
d K=-I d l+\beta(I K)^{1 / 2} d z+\gamma K d w
$$


where $d z$ and $d w$ are the increments of uncorrelated Wiener processes. We will assume that all risk associated with $d z$ is diversifiable, i.e., $d z$ is uncorrelated with the economy and the stock market. However, dw may be correlated with the market. Note that eqn. (4) combines uncertainty over the amount of effort required to complete a project, uncertainty over the cost of that elfort, and uncertainty over the time the project will take.

\section{The Optimal Investment Rule.}

Given that dw in eqn. (4) may be correlated with the narket, we cannot use the riskfree rate of interest for the discount rate $\mu$ in eqn. (3). We can eliminate $;$ from the problem, however, if $d w$ is spanned by existing assets in the cconomy, i.e-, if in principle one could replicate movements in $d w$ with some other asset or dynamic portfolio of assets. The investment problem can then be solved using contingent claims mekhods. II spanning does not hold, we could instead find an optimal investment rule using dynamic programming, subject to some choice of discount rate $\mu^{\top}$

We will assurne that spanning holds. Let $x$ be the price of an asset or dynamic portfolio of assets perfectly correlated with $w$, so that $d x$ follows:

$$
d x=\alpha_{x} x d t+\sigma_{x} x d w .
$$

By the CAPM, the risk-adjusted expected return on $I$ is $r_{s}=r+\theta \rho_{r m} \sigma_{x}$, where $\theta$ is the market price of risk, and $\rho_{x m}$ is the instantaneous correlation of $x$ with the market portfolio.

The Appendix shows that $F(K)$ must satisfy the following differential equation:

$$
\frac{1}{2} \beta^{2} I K F_{K K}+\frac{1}{2} \gamma^{2} K^{-2} F_{K K}-I F_{K}-\phi \gamma K F_{K}-I=r F
$$

where $\phi \equiv\left(r_{x}-r\right) / \sigma_{x}$. Recall that $r_{x}=r+\theta \rho_{x m} \sigma_{x}$. Thus $\phi=\theta \rho_{x m}$. Since $\theta$ is a economy. wide parameter, the only project-specific paraneter needed to determine $\phi$ is $p_{x m}$, which is equal to the coefficient of correlation between fluctuations in cost and the stock market.

\footnotetext{
${ }^{7}$ Bul wibhout spanning we would have no theory lor delermining the correct discount rate (other than by making assumptions about the risk preferences of masagers or the firm's stockholders). Furthermore, the correst diseount rate need nou be conslant. If $d w$ reflects unpredictable changes in the prices of factors such as labor and raw materials, spanming should hold, at least roughiy.

That $i_{1} \theta=\left(r_{m}-r\right) / \sigma_{m}$, where $r_{m}$ is the expected return on the market, and $\sigma_{m}$ is the standard deviation of that return. If we take the New York Stock Exchange Index as the market, over the period $1920-88, r_{m}-r \approx 08$ and $\sigma_{m} \approx .2$, so $\theta \approx .4$.
} 
Note that eqn. (6) is the Bellman equation for the stochastic dynamic programming problem given by (3), but with $\mu$ replaced by $r$. Because eqn. (6) is linear in $I$, the rate of investment that maximizes $F(K)$ is always equal to either 0 or the maximum rate $k$ :

$$
I= \begin{cases}k & \text { for } \frac{1}{2} \beta^{2} K F_{K K}-F_{K}-1 \geq 0 \\ 0 & \text { otherwise }\end{cases}
$$

Eqn. (6) therefore has a free boundary at a point $K^{*}$, such that $I(t)=k$ when $K \leq K^{*}$ and $I(t)=0$ otherwise. The value of $K^{*}$ must be found as part of the solution for $F(K)$. To determine $F(K)$ and $K^{*}$, we solve (6) subject to the following boundary conditions:

$$
\begin{gathered}
F(0)=V \\
\lim _{K^{\prime} \rightarrow \infty} F(K)=0 \\
\frac{1}{2} \beta^{2} K^{*} F_{K K}\left(K^{*}\right)-F_{K}\left(K^{*}\right)-1=0
\end{gathered}
$$

and $F(K)$ continuous at $K^{*}$. Condition (8) says that at completion, the payoff is $V$. Condition (9) says that when $K$ is very large, the probability is very small that over some finite time it will drop enough to begin the project. Finally, condition (10) follows from (7), and is equivalent to the "smooth pasting" condition that $F_{K}(K)$ be continuous at $K^{*}$.

When $I=0$, eqn. (6) has the following simple analytical solution:

$$
F=a K^{b}
$$

where, to satisfy boundary condition $(9), b$ is the negative root of the quadratic equation $\frac{1}{2} \gamma^{2} b(b-1)-\phi \gamma \delta-r=0$, i.e.,

$$
b=\frac{1}{2}+\frac{\phi}{\gamma}-\frac{1}{2 \gamma} \sqrt{(\gamma+2 \phi)^{2}+8 r}
$$

The parameter $a$ is determined from the remaining boundary conditions, together with $K^{*}$ and the solution for $F(K)$ for $K<K^{*}$. This must be done numerically, which is relatively easy once eqn. (5) has been appropriately transformed. ${ }^{9}$ A family of solutions for $K<K^{*}$

\footnotetext{
When $I=k_{1}$ eqn. (6) has a first-degree singularity at $K=0$. To eliminale this, make the substitution $F(K)=f(y)$, where $y=\log K$. Then $(6)$ becomes:

$$
f_{y y}(y)-f_{y}(y)-\frac{2 k f_{y}(y)}{\beta^{2} k+\gamma^{2} e^{y}}=\frac{2 k+2 r f(y)}{\beta^{2} k e^{-y}+\gamma^{2}}
$$

and boundary conditions (8) to (10) are transformed accordingly.
} 
can be found that satisfy condition (8), but a unique solution, together with the value of $a$, is determined from (10) and the continuity of $F(K)$ at $K^{*}$.

\section{Solution Characteristics.}

The effects of cost uncertainty can be seen by first examining solutions of eqn. (6) for the case of pure technical uncertainty, i.e., $\gamma=0$, and then for the case of pure input cost uncertainty, i.e., $\beta=0$. Afterwards we will return to the general case.

\section{Technical Uncertainty.}

When only technical uncertainty is present, eqn. (6) reduces to:

$$
\frac{1}{2} \beta^{2} I K F_{K K}-I F_{K}-I=r F .
$$

In this case, $K$ can change only when investment is taking place, so if $K>K^{*}$ and the firm is not investing, it never will, and $F(K)=0$. Hezce boundary conditions (8) and (10) remain the sarne, but condition (9) is replaced with $F\left(K^{*}\right)=0$.

When $r=0$, equ. (13) has an analytical solution:

$$
F(K)=V-K+\beta^{2}\left(\frac{V}{2}\right)^{-2 / \beta^{2}}\left(\frac{K}{\beta^{2}+2}\right)^{\left(\beta^{2}+2\right) / \beta^{2}},
$$

and the critical value of $K, K *$, is given by:

$$
K^{*}=\left(1+\frac{1}{2} \beta^{2}\right) V
$$

Eqn. (14) has a simple interpretation. With $r=0, V-K$ would be the value of the investment opportunity were there no possibility of abardoning the project. The last term is the value of the put option, i.e., the option to abandon the project should costs turn out to be much higher than expected. Note that for $\beta>0, K^{*}>V$, and $K^{*}$ is increasing in $\beta$. The mose uncertainty there is, the greater the value of the investment opportunity, and the larger is the maxinum expected cost for which beginning to invest is economical.

When $r>0$, eqn. (13) does not have an analytical solution, but can be solved numerically for different values of $\beta$. To choose values for $\beta$ that are reasonable, we need to relate this 
parameter to the variance of the project's total cost. The Appendix shows that for this case in which $\gamma=0$, the variance of the cost to completion is given by:

$$
\operatorname{Var}(\vec{K})=\left(\frac{\beta^{2}}{2-\beta^{2}}\right) K^{2}
$$

Hence if one standard deviation of a project's cost is 25 percent of the expected cost, $\beta$ would be 0.343 , and if one standard deviation is 50 percent of the expected cost, $\beta$ would be 0.63. Standard deviations of project cost in the range of 25 to 50 percent are not unusual, so we will use these values for $\beta$ in the calculations that follow.

Figure 1 shows $F(K)$ as a function of $K$ for $V=10, k=2, r=.05$, and $\beta=0, .343$, and .63. Observe that $F(K)$ looks like the value of a put option, except that $F(K)=0$ when $K$ exceeds the "exercise" point $K^{*}$. Although $F(K)$ is larger the higher is $\beta$, the effect is greatest for larger values of $K$. Also, the effect of technical uncertainty on the optimal investment rule is moderate; only when $\beta=, 63$ does $K^{*}$ substantially exceed its value lor the certainty case. In fact, for $K^{*}$ to increase by 50 percent (from about 9 to about 13.5 ), a value of $\beta$ close to 1 is required, which in turn implies that the standard deviation of total cost be about 100 percent of the expected cost.

Firlally, Figure 2 shows how $F(K)$ depends on the maximum rate of investment, $k$. (Here, $\beta=.63$.) As in the centainty case, a larger $k$ implies a larger $F(K)$, because the payoff $V$ is expected to be received earlier, and hence is discounted less. Atso, when the investment opportunity is worth more, the critical value $K^{*}$ is larger.

\section{Input Cost Uncertainty,}

With only input cost uncertainty, eqn. (6) becomes:

$$
\frac{1}{2} \gamma^{2} K^{2} F_{K K^{\prime}}-I F_{K}-\phi \gamma K F_{K}-I=r F
$$

This is again subject to boundary conditions (8) and (9), but condition (10) is replaced with $F_{K}\left(K^{*}\right)=-1$. Now $K$ can change whether or not investment is taking place, so like a financial put option, $F(K)>0$ for any finite $K$.

When $\gamma>0$, eqn. (16) has no solution when $r=0$, because then there would be no reason to ever invest. One would always be better off waiting until $K$ fell close to 0 so that 
the net payoff from investing is larger. It would not matter that substantial time might have to pass for this to happen, because net payoffs would not be discounted.

If $I=0, \hat{K}$ is lognormally distributed. Then $\gamma$ can be interpreted as the standard deviation of percentage changes per period (in this case, a year) in $K$. Determining a value for $\gamma$ that is reasonable depends on the makeup of cost; Section 5 shows how this can be done for a specific example. Figure 3 shows numerical solutions of eqn. (16) for $\gamma=0, .2$ and .4. (In each case, $V=10, k=2, r=.05$, and $\phi=0$.) Observe that even when $\gamma$ is 2 , there is a substantial effect on the value of the investment opporiunity (particularly when $K$ is large), and on the critical cutoff $K^{*}$. When $\gamma=.2, K^{*}$ is about half of what it is when $\gamma=0$, so that a correct net present value rule would require the payoff from the investment to be about wice as large as the expected cost before the investment is undertaken. This is similar to the kinds of numerical results obtained by McDonald and Siegel (1986) and Majd and Pindyck (1987) for uncertainty over the payoff to an investment, and shows that the effects of input cost uncertainty can also be quantitalively important.

Figure 4 shows the dependence of $F(K)$ and $K^{*}$ on $\phi$, i.e., on the extent to which fluctuations in $K$ are correlated with the economy and the stock market. Recall that $\phi=$ $\theta_{\rho_{\mathrm{m}}}=\theta_{\rho_{\mathrm{K} m}}$. A reasonable value for $\theta$, the market price of risk, is 0.4 , so we would expect $\phi$ to be less than this, perhaps on the order of .1 to .3. Figure 4 shows $F(K)$ for $\phi=0,3$, and for illustrative purposes, 6. As is clear from this figure, a value of $\phi$ on the order of 1 will have only a negligible effect on $F(K)$ and $K^{*}$. For a value of 3 , however, the effect is large, and reduces $K=$ by around 25 percent compared to $\phi=0$. Thus input cost uncertainty with a large systematic component can have a substantial inpact on the decision to invest.

\section{The General Case.}

The value of the investment opportunity and the critical expected cost $K^{-}$can be found for any combination of $\beta, \gamma$, and $\phi$ by numerically solving eqn. (6) and its associated boundary conditions. Since increases in $\beta$ and $\gamma$ (or $\phi$ ) have opposite efects on $K^{\prime}$, it is useful to determine the net effect for combinations of these parameters.

Table 1 shows $K^{*}$ as a function of both $\beta$ and $\gamma$, for $\phi=0, V=10, k=2$, and $r=, 05$. 
Table 1 - Critical $K^{*}$ as a Function of $\beta$ and $\gamma$.

(Note: $V=10, k=2, r=.05$, and $\phi=0$.)

$\gamma$

\begin{tabular}{|c|cccccc|}
\hline$\beta$ & 0 & 0.1 & 0.2 & 0.3 & 0.4 & 0.5 \\
\hline 0 & 8.9257 & 6.6113 & 4.9463 & 3.7524 & 2.8857 & 2.2559 \\
0.1 & 8.9844 & 6.6504 & 4.9756 & 3.7720 & 2.9016 & 2.2681 \\
0.2 & 9.1309 & 6.7578 & 5.0537 & 3.8330 & 2.9468 & 2.3032 \\
0.3 & 9.3750 & 6.9385 & 5.1855 & 3.9307 & 3.0225 & 2.3608 \\
0.4 & 9.7168 & 7.1875 & 5.3711 & 4.0674 & 3.1274 & 2.4438 \\
0.5 & 10.156 & 7.5098 & 5.6104 & 4.2480 & 3.2617 & 2.5488 \\
0.6 & 10.693 & 7.9053 & 5.8984 & 4.4629 & 3.4277 & 2.6758 \\
0.7 & 11.328 & 8.3691 & 6.2402 & 4.7168 & 3.6230 & 2.8271 \\
0.8 & 22.051 & 8.8965 & 6.6309 & 5.0146 & 3.8477 & 3.0005 \\
0.9 & 12.861 & 9.5020 & 7.0801 & 5.3467 & 4.1016 & 3.1982 \\
1.0 & 13.770 & 10.166 & 7.5732 & 5.7178 & 4.3848 & 3.4180 \\
\hline
\end{tabular}

Note that $K^{*}$ decreases with $\gamma$ and increases with $\beta$, but is much more sensitive to changes in $\gamma$. Whatever the value of $\beta$, a $\gamma$ of 0.5 reduces $K^{*}$ to about a filth of the value it has when $\gamma=0$. Also, this drop in $K^{*}$ would be even larger if there were a systematic component to the input cost uncertainty. Thus for many investments, and particularly for large industrial projects where input costs fluctuate, increasing uncertainty is likely to depress investment. The opposite will be the case only for investments like $\mathrm{R} \& \mathrm{D}$ programs, where technical uncertainty is far more important and $\beta$ could easily exceed 1 .

Table 2 shows $F(K ; \beta, \gamma)$ as a function of $\beta$ and $\gamma$ for $K=8.92$, which is the value of $K^{*}$ when $\beta=\gamma=0$. This is the "premium" in tle value of the investment opportunity that results from the two sources of cost uncertainty. Note that this premium is increasing in both $\beta$ and $\gamma$, but is again more sensitive to $\gamma$. Also, if $\gamma$ is large (say, 0.5 ), this premium changes very little when $\beta$ is increased. 
Table $2-F(K)$ as a Function of $\beta$ and $\gamma$.

(Evaluated at $K^{*}$ corresponding to $\beta=\gamma=0$ )

\begin{tabular}{|c|cccccc|}
\hline$\beta$ & 0 & 0.1 & 0.2 & 0.3 & 0.4 & 0.5 \\
\hline 0 & 0 & 1.0877 & 2.1553 & 3.1588 & 4.0535 & 4.8345 \\
0.1 & .1384 & 1.0915 & 2.1596 & 3.1599 & 4.0565 & 4.8371 \\
0.2 & .2026 & 1.0983 & 2.1642 & 3.1670 & 4.0606 & 4.8409 \\
0.3 & .2428 & 1.1149 & 2.1753 & 3.1747 & 4.0692 & 4.8456 \\
0.4 & .3924 & 1.1434 & 2.1956 & 3.1878 & 4.0810 & 4.8595 \\
0.5 & .5199 & 1.1918 & 2.2277 & 3.2146 & 4.0974 & 4.8746 \\
0.6 & .7499 & 1.2650 & 2.2697 & 3.2440 & 4.1240 & 4.8920 \\
0.7 & .9067 & 1.3652 & 2.3280 & 3.2837 & 4.1572 & 4.9184 \\
0.8 & 1.1664 & 1.4942 & 2.3998 & 3.3401 & 4.1978 & 4.9487 \\
0.9 & 1.3606 & 1.6848 & 2.4939 & 3.4024 & 4.2460 & 4.9884 \\
1.0 & 1.6034 & 1.8724 & 2.5996 & 3.4704 & 4.3021 & 5.0323 \\
\hline
\end{tabular}

The use of this model for investment decisions requires estimates of $\beta$ and $\gamma$, and, secondarily, an estimate of $\phi$ or $\rho_{K m}$. This requires estimating confidence intervals around projected cost for each source of uncertainty. To break total cost uncertainty down into technical and input cost components, one can utilize the fact that the frst is independent of time, whereas the variance of cost due to the second component grows linearly with the time horizon. Thus, a value for $\gamma$ is found by estimating the standard deviation of cost $T$ years into the future assuming no investment takes place prior to that time. This estimate, $\sigma_{T}$, could come from experience with construction costs, or from an accounting model of cost combined with variance estimates for individual iuputs. Then, $\hat{\gamma}=\bar{\sigma}_{T} / \sqrt{T}$. Likewise, using eqn. (15) and an initial estimate of expected cost, $K(0)$, a value for $\beta$ car be based on an estimate of the time-independent standard deviation of $\tilde{K}$. In the next section, I illustrate this in the context of a specific example - the decision to build a nuclear power plant. 


\section{Example - The Construction of Nuclear Power Plants.}

We will examine the decision to start or continue building a nuclear power plant in the context of market conditions in late 1982 or 1983 . This was about three years after Three Mile Istand, and was a time of considerable uncertainty over nuclear plant construction costs, which had begun rising sharply. Many utilities faced diffcult decisions whether to go ahead with planned or ongoing construction, and sorne cancelled plants that were well on their way towards completion. ${ }^{10}$ Examining this investment problen will show how the nodel can be used, and provide insight into the evolution of nuclear power in the U.S.

To apply the model, we need estimates of the expectation and variance of the cost of building a kilowatt of nuclear generating capacity, a decomposition of that variance into techrical and factor cost components, the maximum rate of investment, and the value of the unit of capacity. The last two numbers are relatively straightforward. Given the prices of alternative fuels during the early-and mid-1980s, the value of a unit of capacity was about $\$ 2,000$, with Huctuations in real terms within only a $\pm 10 \%$ range. ${ }^{12}$ The actual construction time for nuclear plants varied through time and across plants during the late 1970 s and 1980 s, from 6 to as long as 16 years, but tended to move proportionaliy with realized costs, and increased over the years as (real) costs increased. During the early 1980s, however, estimates of expected construction time were clustered around 10 years, so a good estimate of the maximum rate of investment is 10 percent of expected cost.

To estimate the expectation, variance, and variance decomposition of cost, L use survey data on individual nuclear plant costs published by the Tennessee Valley Authority, and a cross-section regression analysis by Lewis Perl $(1987,1988)$ that cxplains differences in these costs across plants. The TVA obtained quarterly estimates of expected cost for nuclear plants planned or under construction in the U.S. These numbers, published in the TVA's "Costs per Kilowatt Report for U.S. Nuclear Plants," provide data on the expected cost of

\footnotetext{
${ }^{20}$ For example, Virginia Electric Power cancelled its Northanna III and IV units, which were $10 \%$ completed, Public Service of Indiana cancelted Marble Hïl ( $35 \%$ completed), Washington Public Power Supply Systems cancelled four of its five plants ( $5 \%$ to $50 \%$ compieted), and Cleveland Elertric Illuminating cancelled its Zimmer plant, which was more ihan $90 \%$ completed.
}

"All numbers are in 1985 constant dollars. This figure is based on Perl (1987, 1988). 
a kilowatt of generating capacity on a plant-by'plant basis. The variance o[ cost and its decomposition can be estimated from the timeseries and cross-sectional variation of these numbers, using the lact that the variance of cost due to technical uncertainty is independent of time, but the variance due to input cost Huctuations grows with the time horizon.

In any year, expected costs per kilowatt will vary across the 50 to 60 plants in the TVA survey, but part of this variation can be explained by differences in the type of plant, the experience of the contractor, region of the country, etc. Consider the cross-section regression:

$$
\operatorname{COST}_{i t}=a_{0}+a_{1} X_{1 i t}+a_{2} X_{2 i t}+\ldots+\epsilon_{i}
$$

where $\operatorname{COST}_{i t}$ is expected cost for plant $i$ in year $t$, and the $X_{i l}$ 's are a set of explanatory variables. This regression filters out the predictable part of the cross-sectional variation. Then, for plant $i$ in year $t$, an estimator of the variance of cost due to technical uncertainty is the variance of the cross-sectional forecast error for $\mathrm{COST}_{\text {it }}$ from the regression equation (17), given the values of $X_{1 \text { it }}, X_{2 i t,}$ etc., that apply to plant $i$.

A lower bound on this variance is the (squared) standard error of the regression; this would be the variance of the forecast error if, for plant $i, X_{\text {kil }}$ for each $k$ were equal to its cross-sectional mean. In general, the $X_{\mathrm{tit}}$ 's for any plant will differ from the means, so the variance of the forecast error will exceed the squared standard error of the regression. (The reason is that the true coefficients $a_{1}, a_{2}$, etc., are unknown, and only estimated.) An upper bound on the variance of the forecast error is the cross-sectional sample variance of COST itHence I consider values of $\beta$ in eqn. (6) that correspond to forecast error variances ranging from the squared standard error of the regression to the sample variance.

Peri $(1987,1988)$ ran such regressions in logarithmic form for $1977-1985$, using the TVA data on COST for the last quarter of each year, with up to ten explanatory variables. ${ }^{12}$ I infer values of $\beta$ from his results, using the 1982 data and regression. Converting to levets, the

\footnotetext{
${ }^{12} \mathrm{He}$ regresed the $\log$ of COST (in constant 1985 dollars) against a sel of variables that included the $\log$ of the real wage, the log of the net design electric tating (reffecting the scale of the plant), the log of the experience of the architect/engineer (mesured in number of planis designed), and dummy variables for the region of the country, for the type of rock foundation, for whether the plant was the first or subsequent built by the utility, for whether it was a boiling water resctor, for whether the utility served as itte awn construction manager, and for whether the plant had a complex cooling tower. Oniy variables that were statistically significant were retained, so regressions for some years included only a subset of the abave.
} 
mean expected cost for that year was $\$ 1435$ per kilowatt, with a standard error of regression of 17 percent. This is a lower bound on the standard deviation of the cross-sectional forecast error, and using eqn. (15), implies $\beta=.24 .^{13}$ The upper bound is the sample standard deviation, which for 1982 was 46 percent of expected cost, and corresponds to $\beta=.59$.

Next. I estimate the variance due to input cost uncertainty by fitting the annual time series fot mean expected cost to a geometric random walk. The drift and standard deviation of percentage changes in mean expected cost are 12 and .06 respectively for 1977-1985, and .11 and .07 for 1977-1982. Since I consider decisions at the end of 1982 , I use the latter numbers. However, an estimate of the drift based on six years of data (1977-82) is very imprecise, and an expected real rate of increase of mean cost of 5 percent per year would have been reasonable at the time. This would yield an estimated standard deviation of .20 , so I take .07 to .20 as a reasonable range for $\gamma$ in eqn. (6). $\Lambda$ lso, most input cost uncertairty was due to continual and unpredictable regulatory change, rather than factor price fluctuations. Since this is largely uncorrelated with the economy, I set $\phi=0$.

Table 3 shows solutions for $\beta=0, .24$, and .59 , and $\gamma=0, .07$, and .20. In each case, $V=\$ 2000$ per kilowatt, $k=\$ 144$ per year $(10 \%$ of the $\$ 1435$ mean expected cost in 1982$)$, $\phi=0$, and $r=.045 .^{14}$ The table shows the critical cost $K^{*}$, and the value of the utility's investment option (per kilowatt) for an actual expected cost equal to the mean of $\$ 1435$.

Observe that absent input cost uncertainty $(\gamma=0), K^{*}$ ranges from $\$ 1609$ to $\$ 1881$, so that these investments would have been largely economical. (Technical uncertainty increases $K^{*}$ by 4 to 21 percent compared to its value of $\$ 1550$ when $\beta=\gamma=0$.) But input cost uncertainty lowers $K^{-}$considerably, making the average plant nneconomical. Even for $\gamma=.07$, in most cases it would have been preferable to wait and see how regulations (and the expected costs they implied) evolved. And for $\gamma=.20$, it would have been econornical to

\footnotetext{
${ }^{13}$ Note that this accounts for construction experience and movement down the learning curve. For a discussion of the impact of experience on nuelear plant operating costs, see McCabe (1991). McCabe aiso examines technology adoption with uncertain operating cost and argues that utilities buy a mix of technologies in order to teouce the variance of operating cost.

14 The average yield on 3-year and 10-year Treasury bonds in 1982 was $13 \%$. I take the $1970-82$ average rate of intation of $7 \%$ in the PPI and $10 \%$ in the CPJ as estimates of expected inflation, which puts the real risk-free rate at about $3-6 \%$.
} 
Table 3 - Critical Cost per Kilowatt of Capacity at End of 1982.

(Based on $V=\$ 2000$ per Kilowat $t, r=.045, k=\$ 144$ per year, and $\phi=0$. Mean expected cost was $\bar{K}=\$ 1435$ per kilowatt.)

$\gamma$

\begin{tabular}{|c|c|c|c|}
\hline & 0 & .07 & .20 \\
\hline 0 & $K^{*}=1550$ & $K^{*}=1251$ & $K^{*}=867$ \\
& $F(\bar{K})=121$ & $F(\bar{K})=194$ & $F(\bar{K})=465$ \\
\hline .24 & $K^{*}=1609$ & $K^{*}=1260$ & $K^{*}=871$ \\
& $F(\bar{K})=131$ & $F(\bar{K})=201$ & $F(\bar{K})=469$ \\
\hline .59 & $K^{*}=1881$ & $K^{*}=1293$ & $K^{*}=887$ \\
& $F(\bar{K})=215$ & $F(\bar{K})=228$ & $F(\bar{K})=487$ \\
\hline
\end{tabular}

stop construction on plants that were 40 percent complete. ${ }^{15}$ This would seem to justify the decisions that some utilities made at the time to cancel planned or ongoing construction. ${ }^{16}$

The resuits are not very sersitive to the maximum rate of investment, $k$. Taking $\beta=.24$ and $\gamma=.07$, if $k=288$ (so expected construction time is 5 years instead of 10 ), $K^{\text {r* rises to }}$ $\$ 1397$. If $k=96$ (so construction is expected to take 15 years), $K^{*}$ falls to $\$ 1154$. Thus lor a reasonable range of expected construction times, $K^{-}$varies by \pm 10 percent.

These results show that for nuclear power plants, input cost urcertainty matters most for the investment decision, even though there is substantial technical uncertainty. They also show the importance of incorporating both types of uncertainty in the analysis, rather than treating them separately. Note from the table that the dependence of $K^{*}$ on $\beta$ is much less

\footnotetext{
${ }^{15}$ This assumes that there is no cost to stopping, and that construction could be resumed in the fulure.

15 The TVA surveys were available to all U.S. utilities, so presumably they could have performed the same analysis.
} 
when $\gamma$ is -07 or 20 than it is when $\gamma$ is zero. So, if one first calculated the change in $K^{*}$ due to, say, a $\beta$ of .59 (holding $\gamma=0$ ), and then the percentage change due to a $\gamma$ of .07 , the result would be a $K^{*}$ of about $\$ 1518$, rather than the correct value of $\$ 1293$.

\section{Extensions of the Model.}

This section shows how the model can be extended to account for uncertainty over the future value of the completed project, and to allow for more general processes for $K(t)$.

Uncertainty over the Value of the Completed Project.

Suppose the evolution of $K$ is again given by eqn. (4), but $V$ ajso evoives stochastically:

$$
d V=\alpha_{v} V d t+\sigma_{v} V d z_{\mathrm{w}}
$$

where $d z_{v}$ is assumed to be uncorrelated with $d z$ or $d w$. Thus future values of $V$ are $\log$ normally distributed, and since the project takes time to complete, the payoft is always uncertain. For simplicity, we will assume that all risk is divcrsifiable. Then we can use dynamic programming, discounting with the risk-[ree rate of interest.

The value of the investment opportunity is again given by eqn. (3), but with $V$ now stochastic, and hence replaced by $V(\tilde{T})$. The Beilman equation is:

$$
r F^{\prime}=\max _{I(L)}\left\{-I(t)-I F_{K}+\frac{1}{2} \beta^{2} I K F_{K^{\prime} K}+\frac{1}{2} \gamma^{2} K^{2} F_{K K}+\alpha_{v} V F_{V}+\frac{1}{2} \sigma_{v}^{2} V^{2} F_{V V}\right\}
$$

This is linear in $I$, and eqn. (7) again applies. The optimal rule is to invest whenever $K \leq K^{-}(V)$. Eqn. (19) is an elliptic partial differential equation with a free boundary along the line $K^{*}(V)$. The solution must satisfy the boundary conditions: (j) $F(0, V)=V$, (ii) $\lim _{V \rightarrow 0} F(K, V)=0,(\mathrm{iij}) \lim _{K \rightarrow \infty} F(K, V)=0$, (iv) $\frac{1}{2} \beta^{2} K^{*} F_{K K^{*}}\left(K^{*}, V\right)-F_{K}\left(K^{*}, V\right)-1=0$, and $F(K, V)$ and $F_{K}(K, V)$ continuous at $K(V)$. Condition (ii) reflects the tact that 0 is an absorbing barrier for $V$; the other conditions are interpreted as before.

When $K>K^{*}(V)$, so that $I=0$, eqn. (19) has the following analytical solution:

$$
F(K, V)=m(K / V)^{\omega}
$$

where

$$
\omega=\left(\frac{1}{2}+\frac{\alpha_{v}-\sigma_{v}^{2}}{\gamma^{2}+\sigma_{v}^{2}}\right)\left(1-\sqrt{1+\frac{2 r\left(\gamma^{2}+\sigma_{v}^{2}\right)}{\left(\gamma^{2}+2 \alpha_{v}-\sigma_{v}^{2}\right)^{2}}}\right)
$$


When $K<K^{*}(V)$, use the continuity of $F(K, V)$ and $F_{K}(K, V)$ at $K^{*}$ to eliminate $m$ :

$$
F\left(K^{*}, V\right)=\left(K^{*} / \omega\right) F_{K}\left(K^{*}, V\right)
$$

Equ. (19) together with conditions (i) and (22) can be solved numerically using a finite difference method. The boundary, $K^{*}(V)$, is found simultaneously with $F(K, V)$.

\section{Generalizing the Process for $K(t)$.}

We imposed restrictions on $K(t)$ that resulted in a simple investment rule and let us ciearly differentiate between two types of cost uncertainty. We let $K(t)$ follow:

$$
d K=-I d t+\beta K(I / K)^{\alpha} d z
$$

with $\alpha=0$ or $\frac{I}{2}$. Now suppose $0<\alpha<\frac{1}{2}$. We will again assume that $d z$ is diversifiable, and that $V$ is fixed and certain. Then the Bellman equation is:

$$
\tau F=\max _{I(1)}\left\{-I(t)-I F_{K}+\frac{1}{2} \beta^{2} I^{2 \alpha} K^{2(1-\alpha)} F_{K K}\right\}
$$

Maximizing with respect to $I$ gives the optimal investment rule in terms of $F(K)$ :

$$
I^{*}(K)=\left[\frac{\alpha \beta^{2} K^{2(1-\infty)} F_{K K}}{1+F_{K}}\right]^{1 /(1-2 \alpha)}
$$

Substituting $I^{*}(K)$ into (24) yields the following nonlinear differentiai equation for $F(K)$ :

$$
r F=1+F_{K}-\left(\alpha \beta^{2} K^{2-2 \alpha} F_{K K}\right)^{1 /(1-2 \alpha)}\left(1+F_{K}\right)^{-2 \alpha /(1-2 a)}
$$

To find $F(K)$, (26) must be solved (numerically) subject to conditions (8) and (9).

Eqn. (26) has solutions for which $-1<F_{K} \leq 0$ and $F_{K K}>0 .{ }^{17}$ Note from equ. (25) that $I \rightarrow 0$ as $K \rightarrow 0$, so for smatl $K, I$ falls as the net payoff $V-K$ rises. This is the opposite of Grossman and Shapiro's (1986) finding that $I$ rises as the net payolf rises when there are decreasing returns to effort. In my model there are constant returns to effort; $I$ falls because the variance of $\tilde{K}$ falls as $K$ falls, so that the shadow value of learning falls.

\footnotetext{
${ }^{17}$ At $K=0, F_{K}$ must be greater than -1 as long as construction takes firite time and the discount rate is positive. Likewise, $F_{K K}$ musl remain firite as $K \rightarrow 0$.
} 


\section{Conclusions.}

The model developed in this paper, as well as such predecessors as Roberts and Weitzman (1981) and Grossman and Shapiro (1986), belong to a broad class of optimal search problems asalyzed by Weitzman (1979). In what he characterized as a "Pandora's box" problem, one must decide how many investment opportunities with uncertain outcomes should be undertaken, and in what order. In this paper, each doliar spent towards completion of a project is a single investment opportunity, and the uncertain outcome is the amount of progress that results. The model developed here is more general in that expected outcomes can evolwe stochastically even when no investment is taking place (input cost uncertainty), but more restrictive in that the order in which dollars are spent is predetermined.

One advantage of this model is that it leads to a simple investment ruie that is relativeiy easy to apply in practice. Also, the restrictions that have been imposed on the process for $K(t)$ aliowed us to clearly differentiate between two types of cost uncertainty. As we have seen in the previous section, some of the restrictive assumptions in the model can be relaxed (e.g., that $V$ is non-stochastic), but at the cost of added computational complexity. Other restrictions can be relaxed as well. For example, we can relax the restriction that technical uncertainty is the same for each phase of the project (i.e., the uncertainty over the first third of a project's anticipated cost is the same as for the last third) by making $\beta$ in eqn. (13) a function of $K$. As long as $\beta(K)$ is a smooth monotonic function, it is reasonably straightforward to obtain numerical solutions for $F(K)$.

The sources and amounts of cost uncertainty will vary greatly across different projects. However, based on the ranges of parameter values that would apply to the bulk of large capital invesiments, factor cost uncertainty is likely to be more important than technical uncertainty in terms of its effect on the investment rule and the value of the investment opportunity. We saw that this is clearly the case for investments in nuclear power plants. The opposite may be the case for some R\&D projects. And although we found that $K^{*}$ is not very sensitive to $\beta$, this was based on the assumption, discussed above, that the uncertainty is the same across all phases of the project. Increases in $K^{*}$ may be much larger if much of a project's uncertainty gets resolved during its early phases. 


\section{Appendix}

A. Mean and Variance of $\overline{\mathbf{K}}$.

Here I show that if $K(t)$ follows a controlled diffusion of the form:

$$
d K=-k d t+\beta K(k / K)^{\alpha} d z
$$

then $K(t)$ is inded the expected cost to completion. Let:

$$
M(K)=E_{t}\left[\int_{t}^{\bar{T}} k d \tau \mid K(t)\right],
$$

where $\tilde{T}$ is the first passage time for $K=0$. We will show that $M(K)=K$.

We make use of the fact that the functional $M(K)$ must satisfy the Kolmogorov backward equation corresponding to $(A-I)$ :

$$
\frac{1}{2} \beta^{2} k^{2 \alpha} K^{2-2 \alpha} M_{K K}-k M_{K}+k=0,
$$

subject to the boundary conditions (i) $M(0)=0$ and (ii) $M(\infty)=\infty$. (See Karlin and Taylor (1981), Chapter 15.) Glearly $M(K)=K$ is a solution of (A - 3) and the associated boundary conditions. Now consider a more general solution of the form $M(K)=K+h(K)$, where $h(K)$ is an arbitrary function of $K$, By direct integration,

$$
h_{K}(K)=C \exp \left[\frac{2 K^{2 \alpha-1}}{(2 \alpha-1) \beta^{2} k^{2 \alpha}}\right] .
$$

But since $\lim _{K-\infty} h_{K}(K)=C$, the constant $C$ must equal zero to satisfy boundary condition (ii). Hence $M(K)=K$.

For the case of $\alpha=\frac{1}{2}$ (technical uncertainty), we can also find the variance of the cost to go, i.e.,

$$
\operatorname{Var}(K)=E_{t}\left[\int_{t}^{\bar{\tau}} k d \tau \mid K\right]^{2}-K^{2}(t)
$$

Let $G(K)=E_{\mathrm{t}}\left[\int_{t}^{\bar{T}} k d \tau \mid K\right]^{2}$. Then $G(K)$ must satisfy the following Kolmogorov equation:

$$
\frac{1}{2} \beta^{2} k K G_{K K}-k G_{K}+2 k K=0
$$


subject to the boundary conditions $G(0)=0$ and $G(\infty)=\infty$. (See Karlin and Taylor (1981), page 203.) The solution to $(\mathrm{A}-6)$ is $G(K)=2 K^{2} /\left(2-\beta^{2}\right)$, so the variance is:

$$
\operatorname{Var}(K)=\left(\frac{\beta^{2}}{2-\beta^{2}}\right) K^{2} .
$$

\section{B. Derivation of Equation (6).}

Given a replicating asset or portfolio whose price $x$ follows eqn. (5), we can value the firm's investment opportunity as a contingent clairn. First, denote $\delta \equiv r_{x}-\alpha_{x}$. Now consider the following portfolio: hold the investment opportunity, worth $F(K)$, and sell short $n$ units of the asset with price $x$. The value of this portfolio is then $\Phi=F(K) \rightarrow n x$, and the instantaneous change in this value is $d \Phi=d F-n d x$. Since the expected rate of growth of $z$ is $a_{x}<r_{x}$, the short position will require a payment stream over time at the rate $n\left(r_{x}-\alpha_{x}\right) x=n \delta x$. Also, insofar as investment is taking place, holding the investment opportunity implies a payment stream $I(t)$. Thus over an interval $d t$, the total return on the portfolio is $d F-\pi d x-\pi \delta x d t-I(t) d t$.

Next, using Ito's Lemma, write $d F$ as;

$$
\begin{aligned}
d F & =F_{K} d K+\frac{1}{2} F_{K K}(d K)^{2} \\
& =-\int F_{K} d t+\beta(I K)^{1 / 2} F_{K} d z+\gamma K F_{K} d w+\frac{1}{2} \beta^{2} I K F_{K K} d t+\frac{1}{2} \gamma^{2} K^{2} F_{K K} d t
\end{aligned}
$$

Substituting (5) for $d x$, the total return on the portfolio over an interval $d t$ is therefore:

$$
\begin{aligned}
-I F_{K} d t & +\beta(I K)^{1 / 2} F_{K} d z+\gamma K F_{K} d w+\frac{1}{2} \beta^{2} I K F_{K K} d t+\frac{1}{2} \gamma^{2} K^{2} F_{K K} d t \\
& -n \alpha_{x} x d t-n \sigma_{x} x d w-n \delta z d t-I d t .
\end{aligned}
$$

By setting $n=\gamma K F_{K} / \sigma_{x} x$, we can eliminate the terms in du, and thereby renove nondiversifiable risk from the portfolio. With $n$ chosen this way, the only risk the portfolio carries is diversifiable, and hence the expected rate of return on the portfolio must be the risk-free rate, $r$. Using this value of $n$ and equating the expected portfolio return to $r(F-n x) d t$ yields equation (6) for $F(K)$. 


\section{FIGURE I - TECHNICAL UNCERTAINTY}

Shows value of investment opportunity, $F(K)$, as function of expected cost to completion, $K$, for $\beta=0$, .343 , and .63 , where 8 describes degree of technical uncertainty. Other parameter values are $\mathrm{V}=10$, $k=2, r=.05$, and $\gamma=\phi=0$. Intersection of $F(K)$ with $K$ axis gives critical expected cost $K^{*}$.

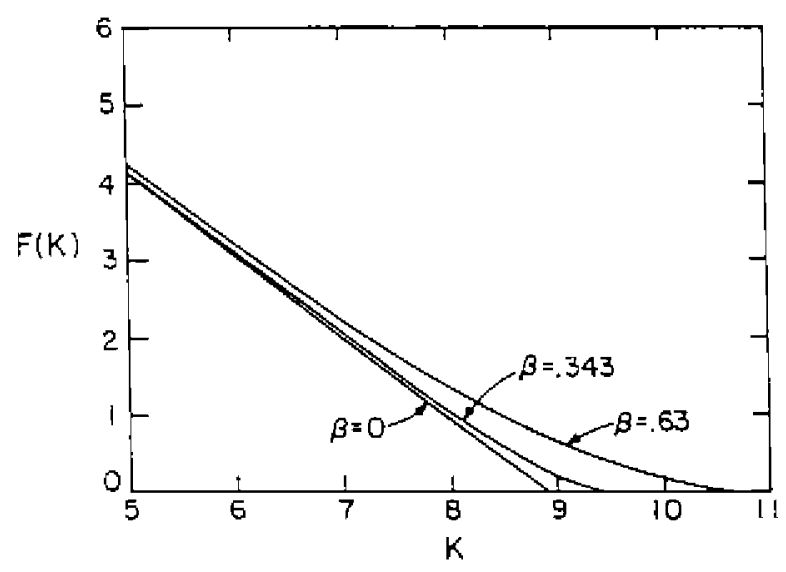

\section{FIGURE 2 - CHANGES IN MAXIMUM RATE OF INVESTMENT}

Shows value of investment opportunity as function of expected cost to completion for three values of maximum ate of iwestment: $\mathfrak{k}=1,2$ and 10 . Only technical uncertainty is present $(\beta=.63, \gamma=\phi$ $=0$ ). Other paraneter values are $\mathrm{V}=10$, and $\mathbf{r}=.05$.

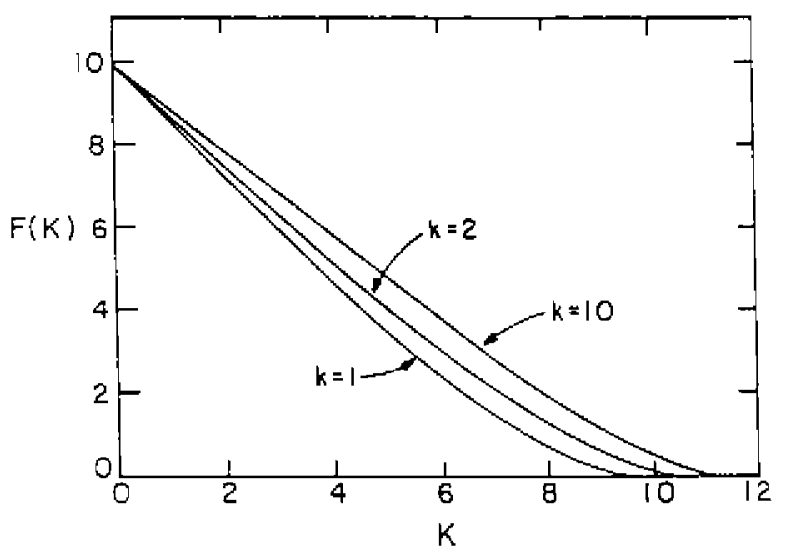




\section{EIGURE 3-INPUT COST UNCERTAINTY}

Shows value of investment opporturity as function of expected cost to completion, and critical expected cost $\mathrm{K}^{*}$, for $\gamma=0,2$, and .4, where $\gamma$ is annual standard deviation of percentage changes in cost due to input cost fluctuations. Other parameter values are $\mathrm{V}=10, \mathrm{k}=2, \mathrm{r}=.05, \beta=0$, and $\phi=0$.

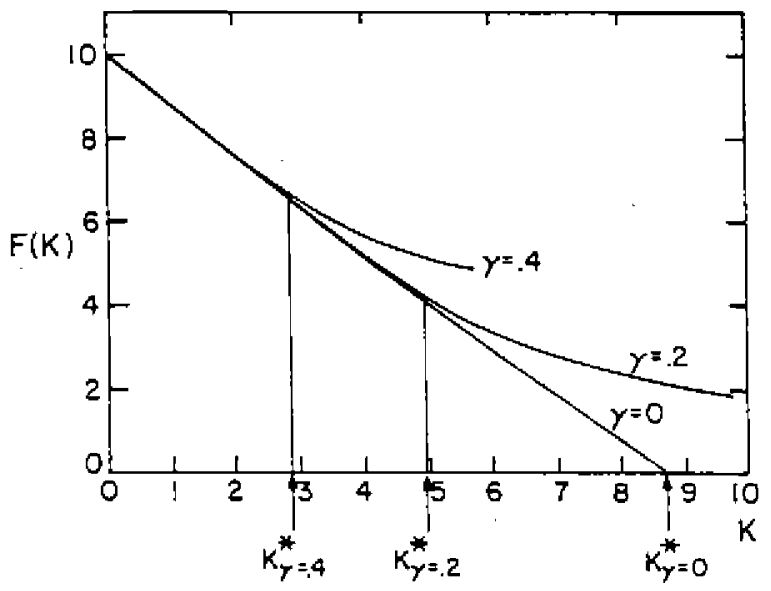

EIGURE 4 - INPUT COST UNCERTAINTY WITH SYSTEMATIC RISK

Shows value of investment opportunity as function of expected cost to completion, and critical expected cost $\mathrm{K}^{*}$, for $\phi=0,3$, and .6 . Only input cost uncertainty is present $(\gamma=.2, \beta=0)$. Other parameter values are $\mathrm{V}=10, k=2$, and $\mathrm{r}=.05$.

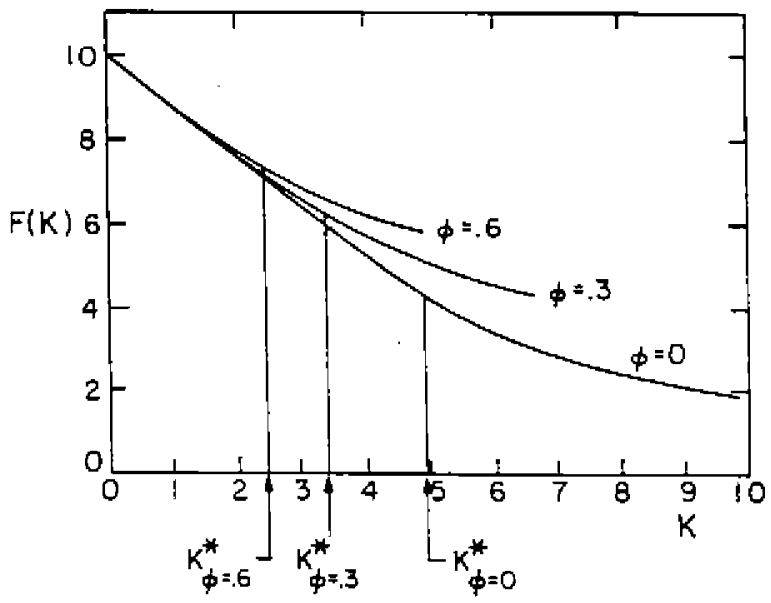




\section{References}

Baldwin, Cariss $Y_{\eta}$ "Optimal Sequential Investment when Capital is Not Readily Reversible," Journal of Finance, June 1982, 37, 763-82.

Bernanke, Beu S., "Irreversibility, Uncertainty, and Cyclical Investment," Quarterly Jousnal of Economics, Feb. 1983, 98, 85-106.

Dixit, Avinash, "Investment and Hysteresis," Journal of Economic Perspectives, Winter 1992, 6, 107-132.

Grossman, Gene M., and Carl Shapiro, "Optimal Dynamic RkD Programs," Rand Journal of Economics, Winter 1986, 17, 581-93.

Karlir, Samuel, and Howard M. Taylor, A Second Course in Stochastic Processes, New York: Academic Press, 1981.

Mackie-Mason, Jefrey K., "Sequential Investment Decisions with Asymmetric Learning," NBER. Working Paper, September 1991.

Majd, Saman, and Robert 5. Pindyck, "Time to Build, Option Value, and Investment Decisions, Journal of Financial Economics, March 1987, 18, 7-27.

Majd, Saman, and Robert S. Pindyck, "The Learning Curve and Optimal Production under Uncettainty," RAND Journal of Econonics, Autumn 1989, 20, 331-343.

McCabe, Mark J., "Industrial Structure and Technological Change in the Nuclear Power Industry" Ph.D. thesis, M.I.T., Sept. 1991.

McDonald, Robert, and Daniel R. Siegel, "The Value of Waiting to Invest," Quarterly Journal of Economics, Nov. 1986, 101, 707-728.

Myers, Stewart C., and Saman Majd, "Calculating Abandonment Value Using Option Pricing Theory, M.I.T. Sloan School of Management Working Paper 1462-83, Jan. 1984.

Perl, Lewis J., Testimony before Public Utility Commission of Texas on behalf of Gulf States Utilities Co., Docket Nos. 7195 and 6755, April 13, 1987.

Peri, Lewis J., Testimony before City Council of City of New Orleans on behalf of Louisiana Power \& Light Co., Application CD-86-11, Jan. 15, 1988.

Pindyck, Robert S., "Irreversibility, Uncertainty, and Investment," Journal of Economic Literature, September 1991, 29, 1110-1152. 
Roberts, Kevin, and Martin L. Weitzman, "Funding Criteria for Research, Development, and Exploration Projects," Econometrica, 1981, 49, 1261-1288.

Tennessee Valley Authority, "Cost per Kilowatt Report for U.S. Nuclear Plants," quarterly surveys, 1977 to 1985 .

Weitzman, Martin, "Optimal Search for the Best Alternative," Econometrica, May 1979, 47, 641-54.

Weitzman, Martin, Whitney Newey, and Michael Rabin, "Sequential R\&D Strategy for Synfuels, ${ }^{n}$ Bell Journal of Economics, 1981, 12, 574-590.

Zeira, Joseph, "Investment as a Process of Search," Journal of Political Economy, February $1987,95,204-210$. 